\title{
MÉTODO FUZZY PARA LA IDENTIFICACIÓN DE ÁREAS POTENCIALES PARA LA PRODUCCIÓN DE LEÑA. APLICACIÓN DE CASO: COMUNA DE EMPEDRADO, REGIÓN DEL MAULE, CHILE
}

\author{
Roberto Garfias Salinas, Miguel Castillo Soto \\ y Adolfo Yáñez Leiva \\ Facultad de de Ciencias Forestales y Conservación de la Naturaleza. \\ Universidad de Chile. Casilla 9206, Santiago de Chile \\ rgarfias@uchile.cl
}

\begin{abstract}
Resumen: Utilizando criterios ambientales y territoriales se identificaron 12.828,6 hectáreas donde se pueden establecer plantaciones dendroenergéticas en la Comuna de Empedrado, Región del Maule, Chile. Las especies forestales propuestas fueron Acacia dealbata, Acacia melanoxylon, Chamaecytisus proliferus ssp. palmensis, Eucalyptus camaldulensis y Robinia pseudoacacia. Se elaboraron modelos de capacidad de acogida del territorio para cada especie con el fin de identificar áreas de alta, media y baja aptitud. La aptitud del terreno para recibir plantaciones dendroenergéticas se evaluó integrando la opinión de expertos en plantaciones forestales y dendroenergía, la información cartográfica y digital disponible de Empedrado y los requerimientos ambientales de las especies. Nueve variables ambientales fueron jerarquizadas, representadas espacialmente y transformadas a una escala común utilizando sistemas de información geográfica (SIG). Al comparar estos modelos Chamaecytisus proliferus posee mayor aptitud en un 70,3\% de la superficie disponible mientras que Robinia pseudoacacia no aparece como recomendable.
\end{abstract}

Palabras clave: Maule, Chile, producción de leña, Lógica Borrosa, Sistema de Información Geográfica.

Abstract: Potential areas for establishing wood energy plantations in the Empedrado municipality, Maule region, were identified using environ-

* Recibido: 27-05-11. Aceptado: 2-11-12. 
mental and territorial criteria. For this purpose, five forest species were proposed, Acacia dealbata, Acacia melanoxylon, Chamaecytisus proliferus ssp. palmensis, Eucalyptus camaldulensis and Robinia pseudoacacia. Suitability land models for wood energy plantations for each propose species were elaborated, in order to quantify and locate areas of high, medium and low suitability. The suitability of the land was evaluated integrating the opinion of experts in forest plantations and wood energy add cartographic and digital information available of the Empedrado municipality, besides the environmental requirements of the species. Nine environmental variables were hierarchized, spatially represented and transformed into a common scale by using Geographic Information Sistem (GIS). When comparing these models the Chamaecytisus proliferus is the species with the highest suitability in $70.3 \%$ of the surface available, whereas Robinia pseudoacacia appears as not recommendable.

Keywords: Maule, Chile, fuelwood production, Fuzzy Logic, GIS.

\section{Introducción}

Existe la necesidad creciente de ampliar la matriz energética para la demanda de bienes y servicios, especialmente en países donde la disponibilidad de recursos naturales factibles de utilizar para este propósito, así lo permite. A pesar que actualmente las principales fuentes de energía provienen de los combustibles fósiles como el petróleo y el gas natural, la leña sigue representando un porcentaje importante del consumo energético en Chile, especialmente en los hogares urbanos y rurales del sur del país, debido principalmente a su bajo precio de mercado, alto poder calorífico y su fácil accesibilidad. Del total de leña que se consume en Chile, un gran porcentaje proviene de especies de los diferentes tipos de bosques nativos que crecen en el país. Esta situación ha producido fuertes presiones de extracción, especialmente en los últimos 25 años, poniendo en serio riesgo la existencia y el estado de degradación de los mismos.

El estudio de áreas potenciales para el abastecimiento de biomasa con fines energéticos no es nuevo. No obstante, muchas de estas iniciativas no han prosperado como se esperaba porque entre otros factores, existe el conflicto político y territorial por la competencia en el uso del suelo o la coexistencia de objetivos que no necesariamente llevan a un uso sustentable del espacio geográfico.

Los bosques en Chile aún siguen siendo parte importante del abastecimiento local de leña, y por ello, la normativa chilena ha efectuado importantes ajustes para que 
esta actividad tenga un mecanismo legal con fines sustentables. Actualmente los protocolos de certificación, tanto en las prácticas de extracción como en la calidad del producto, y la participación de pequeños propietarios en la actividad productiva, son factores que son relevantes en la planificación del territorio para producción de leña.

Por tratarse en muchos casos de áreas locales en donde se presentan las condiciones para su extracción, esta investigación aborda como estudio de caso la Comuna de Empedrado, localizada en la Región del Maule de Chile Central, y que se estima como un área representativa para el diagnóstico extendido de otras áreas potenciales. Se considera este territorio por reunir las condiciones necesarias para proponer una zonificación basada en una nueva metodología de manejo de información geográfica, y que corresponde al establecimiento de umbrales o límites geográficos basados en reglas de decisión respecto a los gradientes o límites geográficos de aprovechamiento para distintas especies forestales que son recomendadas por la normativa chilena para la producción de leña.

Un paso clave en el establecimiento de estos límites es la construcción de una base geográfica de datos que permita un posterior análisis y gestión para aquellos profesionales encargados de ejecutar y tomar decisiones respecto al uso de información con fines de extracción de leña. Para ello, se recomienda utilizar los Sistemas de Información Geográfica (SIG), cuyas herramientas que facilitan el análisis conjunto de las variables territoriales que el usuario considera necesarias para la obtención de criterios espaciales de zonificación. Normalmente cuando de trata de la inclusión de un set de factores que condicionan directamente la aptitud de un territorio, se utiliza la Evaluación Multicriterio (EMC) para ayudar en procesos de análisis espacial y obtención de resultados acordes al cumplimiento de cada uno de los criterios aplicados (Barredo, 1996), y en la validación de los mismos basada en la revisión permanente de las variables consideradas y métodos aplicados para la construcción de áreas potenciales.

En esta investigación, se aborda conjuntamente la EMC junto con las herramientas otorgadas por la lógica fuzzy orientada a establecer gradientes o umbrales para las variables consideradas en el análisis. La opinión de expertos, la calibración de los modelos de capacidad de acogida para cada especie y el ajuste de criterios, permiten entonces generar resultados que para este propósito, se orienta a la identificación de áreas potenciales para la producción de leña. En su metodología se exponen las distintas etapas y criterios utilizados, basado siempre, en las capacidades reales que presentan las especies en términos de su disponibilidad y localización. 


\section{Metodología}

\section{1. Área de estudio}

El área de estudio (figura 1) corresponde a la Comuna de Empedrado (Figura $\mathrm{N}^{\circ}$ 1), que se encuentra ubicada en la Provincia de Talca, Región del Maule, Chile, a $72^{\circ}$ $21^{\prime} 0^{\prime \prime}$ O y $35^{\circ} 37^{\prime} 30^{\prime \prime}$ S (IGM, 2004).

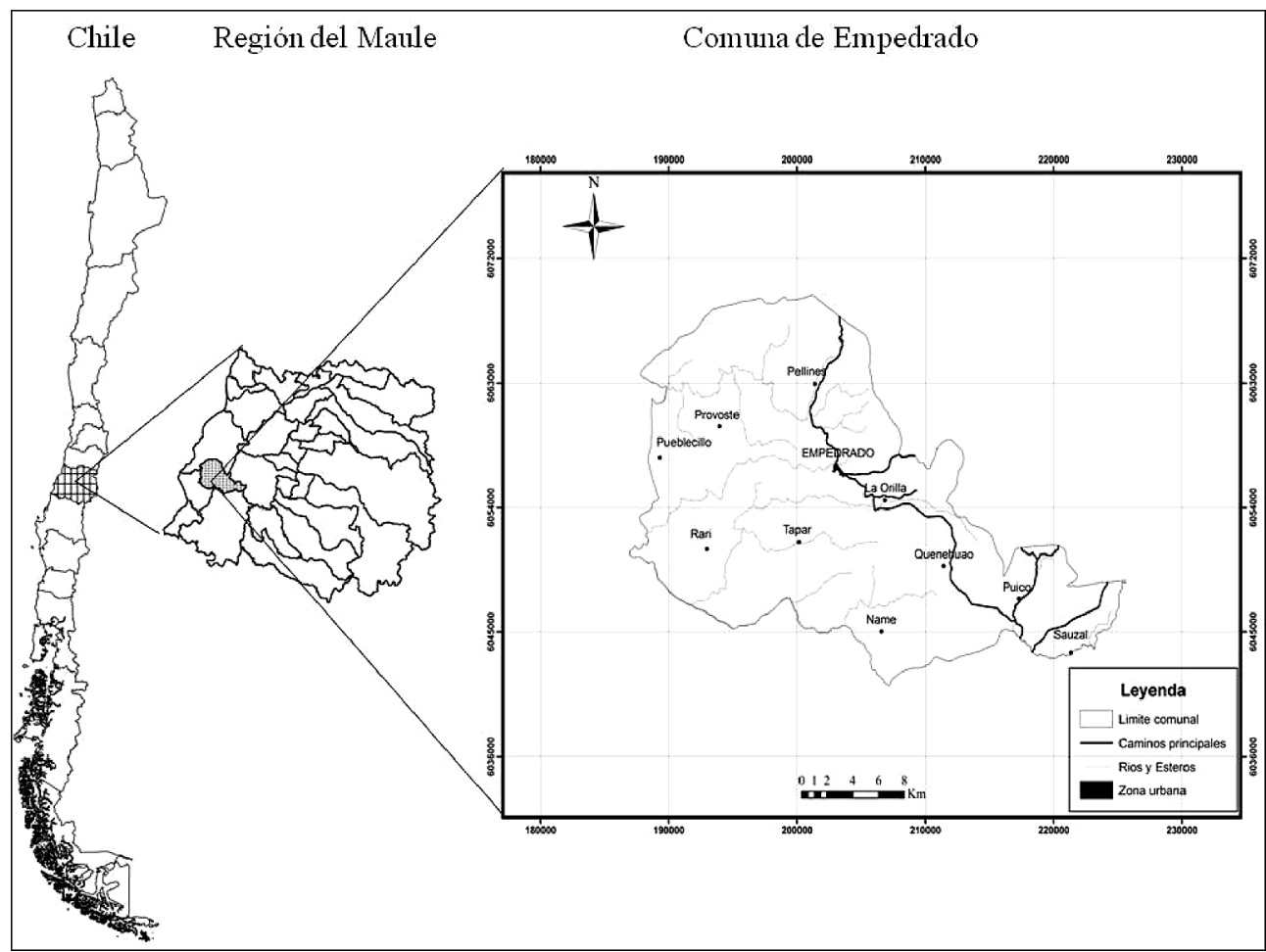

Figura 1. Área de estudio.

\subsection{Base Cartográfica}

Como cartografía base se utilizaron mosaicos IREN-CORFO (1987) a escala 1:20.000, el Plano Digital de la Comuna de Empedrado e información física y ambiental digitalizada, perteneciente al Departamento de Desarrollo Rural de la Municipalidad de Empedrado. La cartografía se rasterizó utilizando un tamaño de pixel de $25 \times 25 \mathrm{~m}$. 


\subsection{Elección de especies y variables}

Después de realizar consultas a expertos y de la revisión bibliográfica, se determinó que las especies con mayor potencial para producción de leña en la Comuna de Empedrado son Acacia dealbata, Acacia melanoxylon, Chamaecytisus proliferus ssp. palmensis, Eucalyptus camaldulensis y Robinia pseudoacacia. Éstas especies cumplen con las características de abastecimiento y sustentabilidad de manejo. Entre las principales cualidades en común, destacan el rápido crecimiento, poseer regeneración vegetativa y ser aptas para diversos propósitos de manejo, especialmente para pequeños propietarios rurales de la zona. Además están presentes en el área de estudio en forma de plantación o asilvestramiento.

Por tratarse de un primer estudio de tipo exploratorio, se consideraron en una primera etapa las variables de tipo ambiental, con énfasis en la topografía. Los aspectos de tipo económico y social pueden ser abordados en la medida de la presentación de los primeros resultados de este estudio. Se seleccionaron nueve variables comúnmente empleadas en la determinación de áreas potenciales, en este caso las que condicionan la existencia y estado de especies forestales para su aprovechamiento en leña, y agrupadas en los criterios de clima, suelo y topografía. Los valores extremos (umbrales) utilizados en cada variable, para cada una de las especies propuestas se presentan en la tabla 1.

Tabla 1. Valores de los requerimientos ambientales para las especies propuestas.

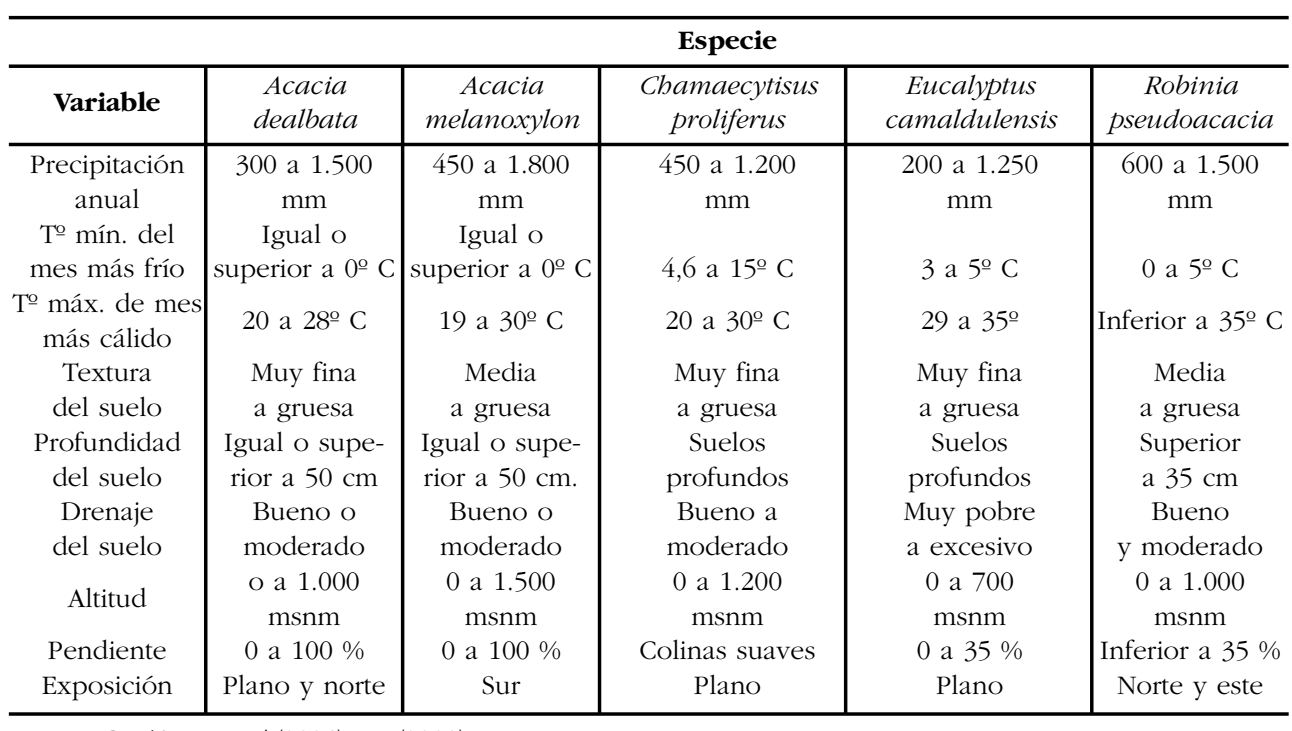

Fuentes: Gestión Forestal (2006), FIA (2000). 


\subsection{Etapas en la elaboración de los modelos de capacidad de acogida del territorio}

La metodología aplicada para obtener los modelos de capacidad de acogida del territorio para cada especie (figura 2) se puede dividir en las siguientes etapas:

- Jerarquización de las variables territoriales.

- Aplicación de la lógica borrosa (Procesos Fuzzy), para las variables consideradas.

- Combinación lineal ponderada de los criterios.

- Zonificación y Restricciones.

- Uso propuesto del suelo.

\subsection{Jerarquización de las variables}

Utilizando la opinión de expertos recogida a través del Método Delphi, las variables consideradas en el estudio fueron jerarquizadas según su nivel de importancia. Un Delphi consiste en la selección de un grupo de expertos a los que se les pregunta su opinión sobre cuestiones referidas a acontecimientos del futuro. Las estimaciones de los expertos se realizan en sucesivas rondas, anónimas, con el objeto de tratar de conseguir consenso, pero con la máxima autonomía por parte de los participantes (Astigarraga, 2004). No hay forma de determinar el número óptimo de expertos para participar en una encuesta delphi. Dependerá del tema, los objetivos del estudio y recursos con que se cuente (Konow y Pérez, 1990).

En este estudio se confeccionó una lista de 12 expertos de distintas instituciones públicas y privadas sobre plantaciones forestales y especies con fines para producción de leña, a los cuales se les entregó un documento por medio de entrevista personal o e-mail, que contenía un resumen donde se explicaban claramente los objetivos del estudio, además de un formulario con los criterios, variables y categorías a evaluar para cada una de las especies. En este formulario los expertos consultados asignaron puntajes normalizados entre 0 y 100 a los criterios y variables, y puntuaciones entre 1 y 10 para las categorías. Además cada experto tuvo la opción de incluir sus recomendaciones respecto al formulario.

Para determinar los niveles de importancia de los criterios, variables y categorías, se utilizó la media aritmética. Al mismo tiempo se utilizaron como medidas de dispersión la desviación estándar y el rango, con el objetivo de calcular las variaciones de las respuestas de los expertos. Los respectivos niveles de importancia obtenidos de los formularios aplicados a los expertos fueron transformados en matrices de comparación entre pares de variables para incluirlos en el módulo de evaluación multicriterio del SIG y así obtener el peso de cada una en el modelo de capacidad de aco- 


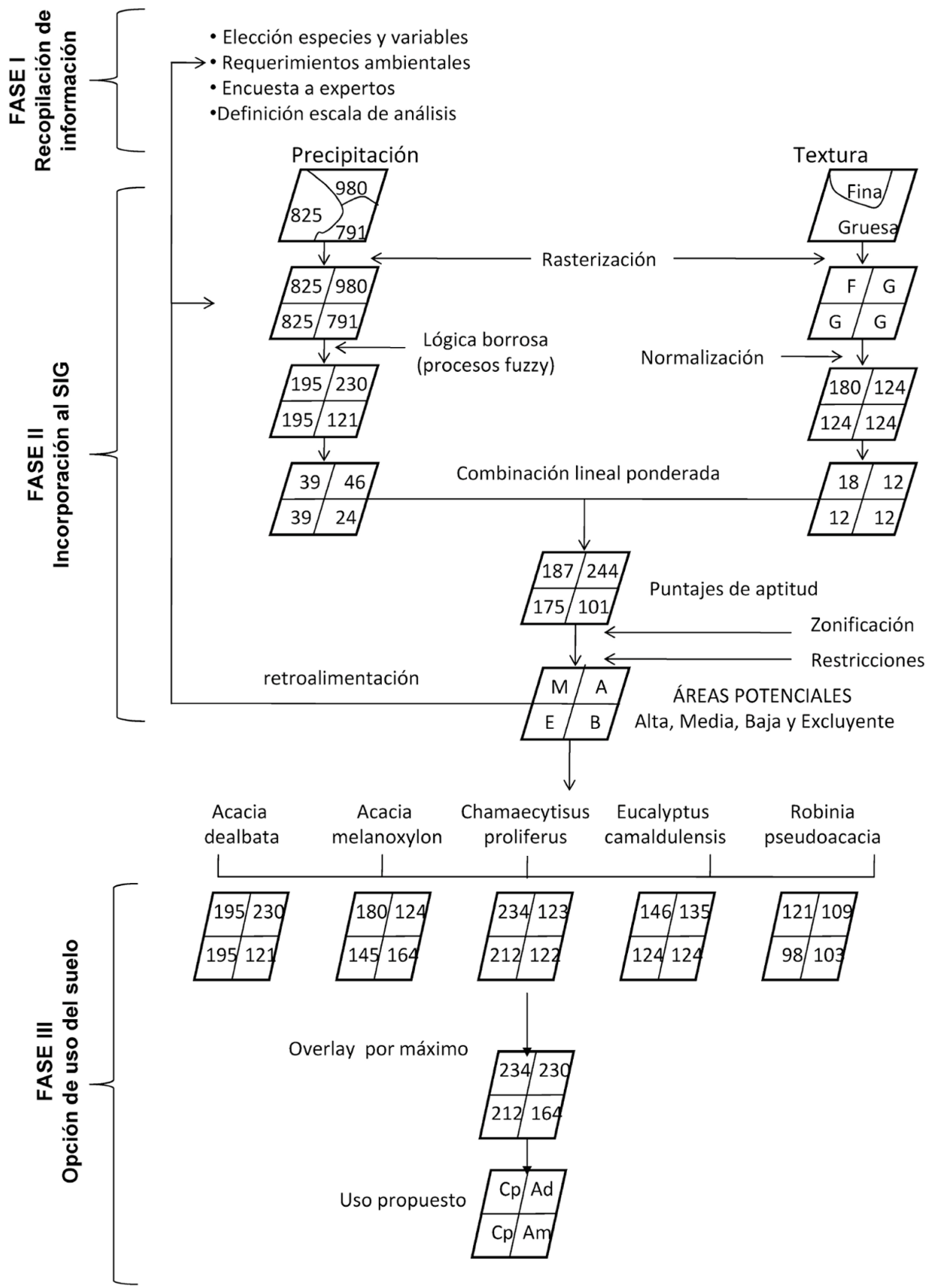

Figura 2. Enfoque metodológico aplicado para los modelos de capacidad de acogida del territorio. 
gida del territorio. Para esto se compararon los rangos (diferencia entre el puntaje máximo y el mínimo) de cada variable, obtenidos de la aplicación del método Delphi. En la matriz de comparación se utilizó una escala continua con valores entre 1/9 y 9 , donde 1/9 significa que la variable en estudio es extremadamente menos importante respecto al conjunto de posibilidades de ponderación respecto al peso de otras variables. Por otro lado, un valor de 9 o cercano, indica que su efecto territorial es extremadamente importante en el efecto de los resultados esperados.

Para eliminar el riesgo de comparaciones incongruentes los SIG calcula la razón de consistencia (que evalúa la discrepancia entre pares de factores para un peso dado) de las comparaciones o CR (consistency ratio). Si este valor es mayor a 0.10 la matriz debe ser reevaluada pues existen comparaciones inadecuadas o incongruentes.

\subsection{Aplicación de la Lógica Borrosa (procesos Fuzzy)}

Las variables, representadas espacialmente en formato raster, se encontraban en distintas escalas dimensionales, por lo que fue necesario estandarizarlas a una unidad común. Para esto se utilizaron funciones de pertenencia (Bellman y Zadeh, 1970; Bonham-Carter, 1994), que oscilan entre 0 y 1 ó 0 y 255, indicando el grado de membresía o pertenencia de un elemento respecto a un conjunto, y pueden ser lineales, en forma de $J$ o sigmoidales (Burrough, 1989; Chen y Hwang, 1992; Ahmed, 2000; Cox, 2005). Todas estas funciones pueden adoptar tendencias gráficas crecientes, decrecientes o simétricas y quedan definidas a través de parámetros o puntos de control $(a, b, c$ y $d)$ que corresponden a los valores extremos y óptimos de las variables
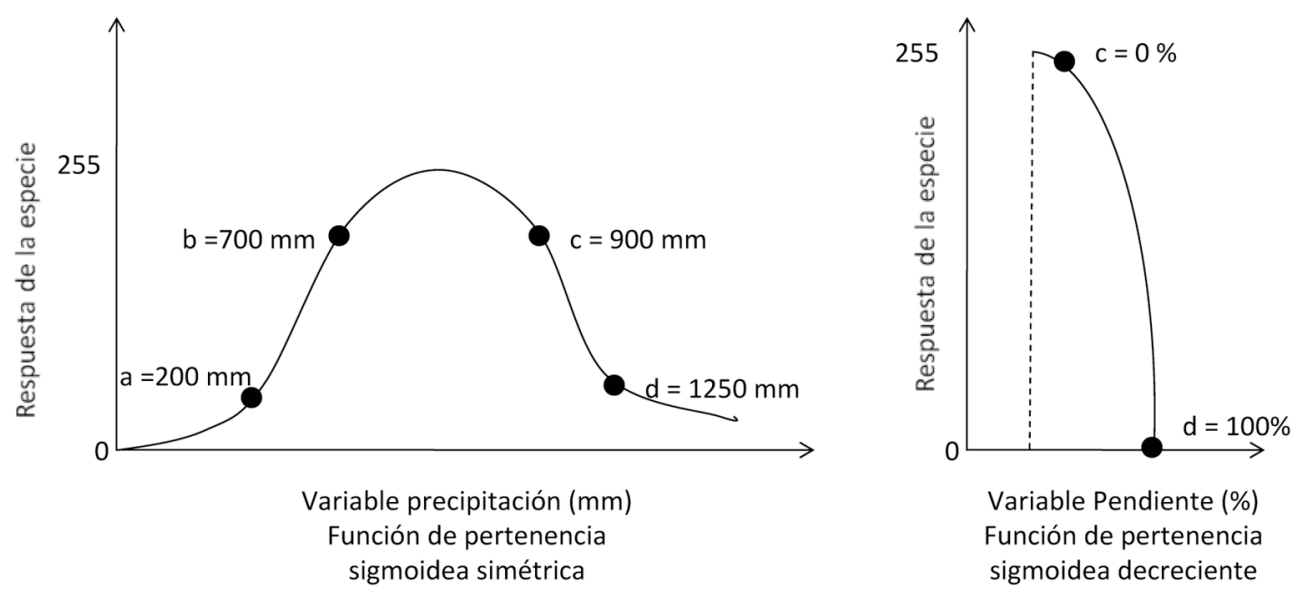

Figura 3. Ejemplos de funciones de pertenencia utilizadas en algunas variables del estudio. 
(Dubois y Prado, 1979; Gill y Bector, 1997; Duprey y Taheri, 2010), obtenidos del análisis de los requerimientos ambientales para cada especie. Específicamente se usaron funciones sigmoideas simétricas y decrecientes (figura 3). Para ocupar todo el espectro gráfico en la imagen raster se consideró el rango 0-255 niveles RGB, donde el valor 0 indica baja o nula capacidad y 255 la más alta capacidad de acogida.

Las variables de drenaje, profundidad y textura del suelo junto con exposición fueron reescaladas debido a que no poseen coberturas con datos de valores continuos, que son las que muestran mejor bondad de ajuste a los métodos multicriterio (Bustillos- Herrera et al, 2007; Voogd, 1993). Se utilizó la opinión de los expertos para identificar los píxeles con mayores diferencias respecto al tipo de dato generado en el rango 0-255. Para reescalar las categorías de esas variables se utilizó la siguiente fórmula:

$$
e_{i j}=\frac{X_{i j}-\operatorname{MinX}_{i j}}{\operatorname{Max}_{i j}-\operatorname{MinX}_{i j}} * 255(\text { Álvarez, 2003) }
$$

Donde:

$e_{i j}=$ Valor normalizado de la categoría i en la variable j. Varía entre cero y 255, que representa la menor y la mayor aptitud del terreno respectivamente.

$X_{i j}=$ Valor de la categoría i en la variable $\mathrm{j}$.

Min y Max = Valores mínimos y máximos de las categorías.

La retroalimentación del sistema consistió en elaborar sucesivos modelos de capacidad de acogida del territorio considerando los criterios de umbrales para cada variable (Burrough, 1989; Ahmed et al, 2000), y ajustando en cada iteración los valores de las capas geográficas utilizadas en el análisis fuzzy, con el propósito de obtener los valores óptimos y las funciones de pertenencia que mejor representan la distribución espacial de cada criterio territorial.

\subsection{Combinación lineal ponderada}

Para generar los Modelos de Capacidad de Acogida del territorio para cada especie, la cobertura digital de cada variable se ponderó por su peso respectivo utilizando la ecuación:

$$
C D A=\sum_{1}^{9} W i * X i
$$

Donde:

$C D A=$ Capacidad de acogida del territorio para recibir plantaciones de la especie correspondiente, expresada en puntajes entre cero y 255. 
$W i=$ Peso de la variable i.

$X i=$ Valor de la variable $\mathrm{i}$.

Como resultado, el SIG entrega una cobertura raster que muestra las superficies factibles de acoger una plantación de la especie correspondiente, en donde cada pixel tiene valores entre cero y 255 . Luego estos valores deben ser reclasificados a las categorías de aptitud correspondiente. Para los modelos de capacidad de acogida se crearon cuatro categorías (alta, media, baja y excluyente) debido a que el nivel de detalle de las distintas capas de información es bajo por la extensión de la zona de estudio.

\subsection{Zonificación y restricciones}

Los rangos de puntuación para clasificar la aptitud se establecieron de forma diferenciada para cada especie utilizando el criterio territorial de segmentación del espacio en unidades llamadas "séptimos" (Julio, 1999). Este método, empleado intensivamente en ordenamiento territorial, que establece que la superficie total de las celdas de alta aptitud debe corresponder a la mitad de la de aptitud media, y esta última, a la mitad de la de aptitud baja. Es decir, aproximadamente 1/7 de la superficie total debe corresponder a una alta aptitud del territorio para recibir plantaciones, los 2/7 de la superficie total corresponden a una aptiud media y los 4/7 finales corresponden a baja aptitud.

El paso siguiente fue incorporar la cobertura raster de las restricciones para extraer las áreas donde no se pueden establecer plantaciones forestales. Posee valores entre 0 y 1 en donde 0 representa un pixel en que es imposible realizar una plantación y 1 indica un terreno que puede recibir plantaciones. Se incorporó al modelo a través de una superposición de imágenes u overlay. Las áreas definidas como excluyentes fueron las cubiertas por plantaciones forestales de interés comercial, y también aquellos territorios cubiertos con bosque nativo. Las zonas urbanas e industriales también fueron excluidas del análisis.

\subsection{Uso propuesto del suelo}

Finalmente, realizando sucesivas operaciones de superposición de mapas, se logró determinar el puntaje máximo para cada pixel del territorio y la especie a la cual correspondía. Como cartografía de síntesis, se elaboraron coberturas raster y vectoriales para cada modelo de capacidad de acogida. 


\section{Resultados}

Al crear los modelos de capacidad de acogida del territorio se identificaron como áreas excluyentes 44.302 hectáreas que representan un 77,5\% del total de la superficie de la Comuna de Empedrado. De éstas, las plantaciones forestales correspondientes a Pinus radiata ocupan 39.797,5 hectáreas representando un 69\% del total de superficie de la comuna, mientras que las de Eucalyptus spp ocupan 71,4 ha con un 0,12\%. El bosque nativo ocupa una superficie de 4.326, 5 ha con un $7,5 \%$ del total comunal.

\section{1. Áreas potenciales para Acacia dealbata}

La capacidad de acogida (CDA) del territorio de la Comuna de Empedrado para recibir plantaciones de Acacia dealbata se calculó como:

$\mathrm{CDA}=0,1688^{*}$ Precipitación $+0,0844^{*} \mathrm{~T}^{\circ}$ mínima $+0,0844^{*} \mathrm{~T}^{\circ}$ máxima + $0,0422 *$ Textura $+0,1480 *$ Profundidad $+0,2108 *$ Drenaje $+0,0844 *$ Altitud + $0,0506^{*}$ Pendiente $+0,1265^{*}$ Expos

La variable drenaje del suelo resultó ser la más importante para determinar áreas potenciales para Acacia dealbata, mientras que la menos importante fue la textura del suelo. Al extraer las áreas excluyentes se obtiene el modelo final de acogida del territorio en el que las áreas con alta aptitud para Acacia dealbata se encuentran en los sectores descritos en la figura 4 .

\section{2. Áreas potenciales para Acacia melanoxylon}

El modelo de capacidad de acogida del territorio para plantaciones de la especie Acacia melanoxylon se calculó en base a la ecuación:

$\mathrm{CDA}=0,1724^{*}$ Precipitación $+0,0862 * \mathrm{~T}^{\circ}$ mínima $+0,0862 * \mathrm{~T}^{\circ}$ máxima + $0,0431^{*}$ Textura $+0,1724^{*}$ Profundidad $+0,1724^{*}$ Drenaje $+0,0862^{*}$ Altitud + $0,0517^{*}$ Pendiente $+0,1293^{*}$ Expos.

Las variables más importantes en la identificación de áreas potenciales para Acacia melanoxylon fueron precipitación, profundidad y drenaje mientras que la menos importante correspondió a textura del suelo. En la figura 5 se puede observar una distribución uniforme de áreas con alta aptitud, excepto en el sector este de la comuna, que corresponde a pequeñas áreas con terrenos de alta aptitud sin embargo, de las cinco especies propuestas, es Acacia melanoxylon la que tiene menor superficie potencial con alta prioridad o aptitud, debido que al aplicar las restricciones al modelo gran parte de estas áreas con alta aptitud se convierten en excluyentes. 


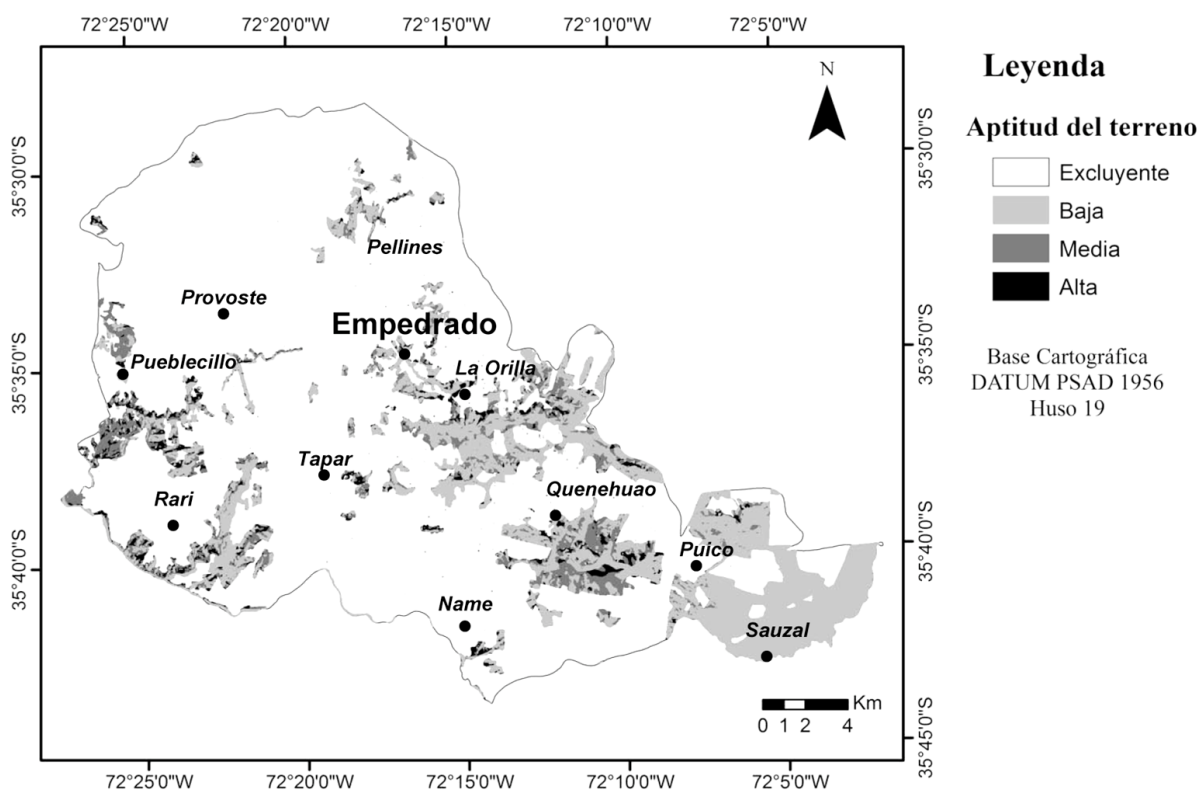

Figura 4. Modelo de capacidad de acogida del territorio para Acacia dealbata.

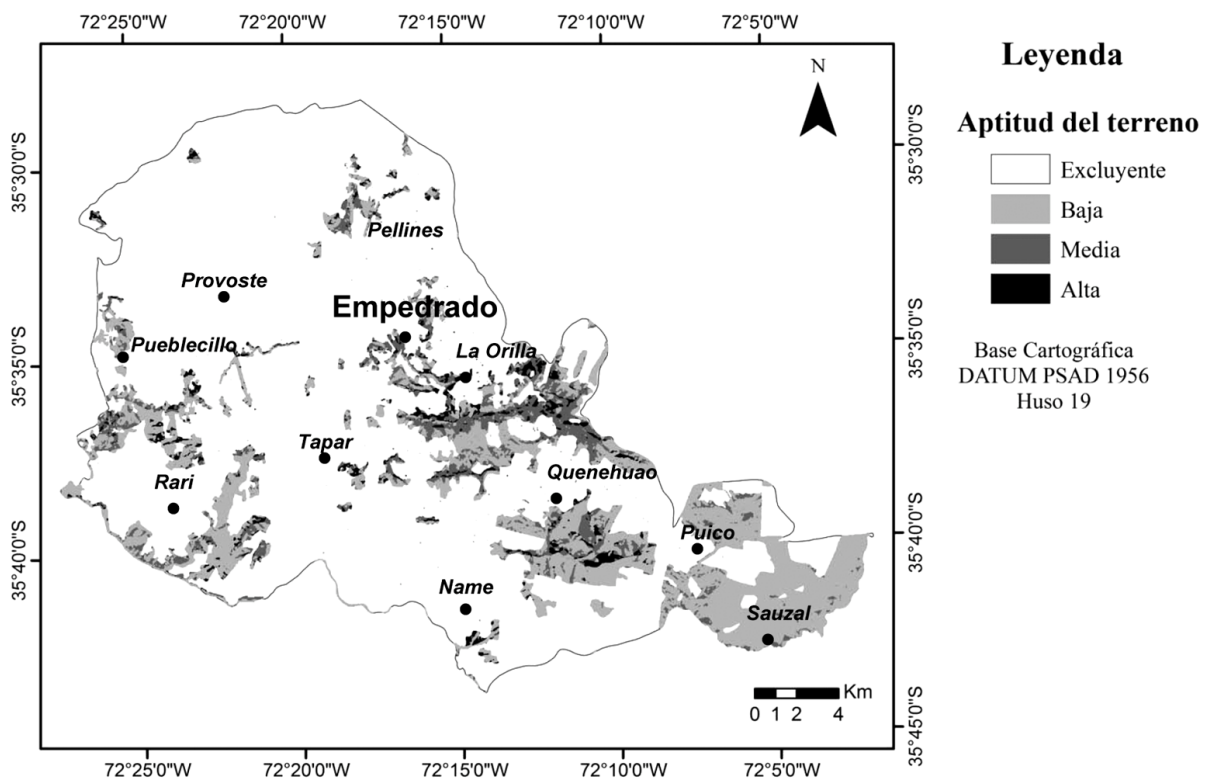

Figura 5. Modelo de capacidad de acogida del territorio para Acacia melanoxylon. 


\section{3. Áreas potenciales para Chamaecytisus proliferus ssp palmensis (tagasaste)}

El modelo de capacidad de acogida del territorio para plantaciones de la especie Chamaecytisus proliferus, se calculó como:

$\mathrm{CDA}=0,1481^{*}$ Precipitación $+0,1852^{*} \mathrm{~T}^{\circ}$ mínima $+0,0741^{*} \mathrm{~T}^{\circ}$ máxima + $0,0741 *$ Textura $+0,1852^{*}$ Profundidad $+0,1111^{*}$ Drenaje $+0,0741^{*}$ Altitud + 0,0370*Pendiente $+0,1111^{*}$ Expos

La temperatura mínima media resultó ser la variable con mayor peso para determinar áreas potenciales para establecer plantaciones de tagasaste, en cambio la variable pendiente resultó ser la menos importante.

En la figura 6 las áreas con mayor aptitud tienden a concentrarse en sectores sur y sur-este de la comuna. Tagasaste fue la especie que obtuvo mayor superficie potencial de alta aptitud para establecer plantaciones dendroenergéticas. Además es la que obtuvo la mayor puntuación de todos los modelos de capacidad de acogida, con 230 puntos.

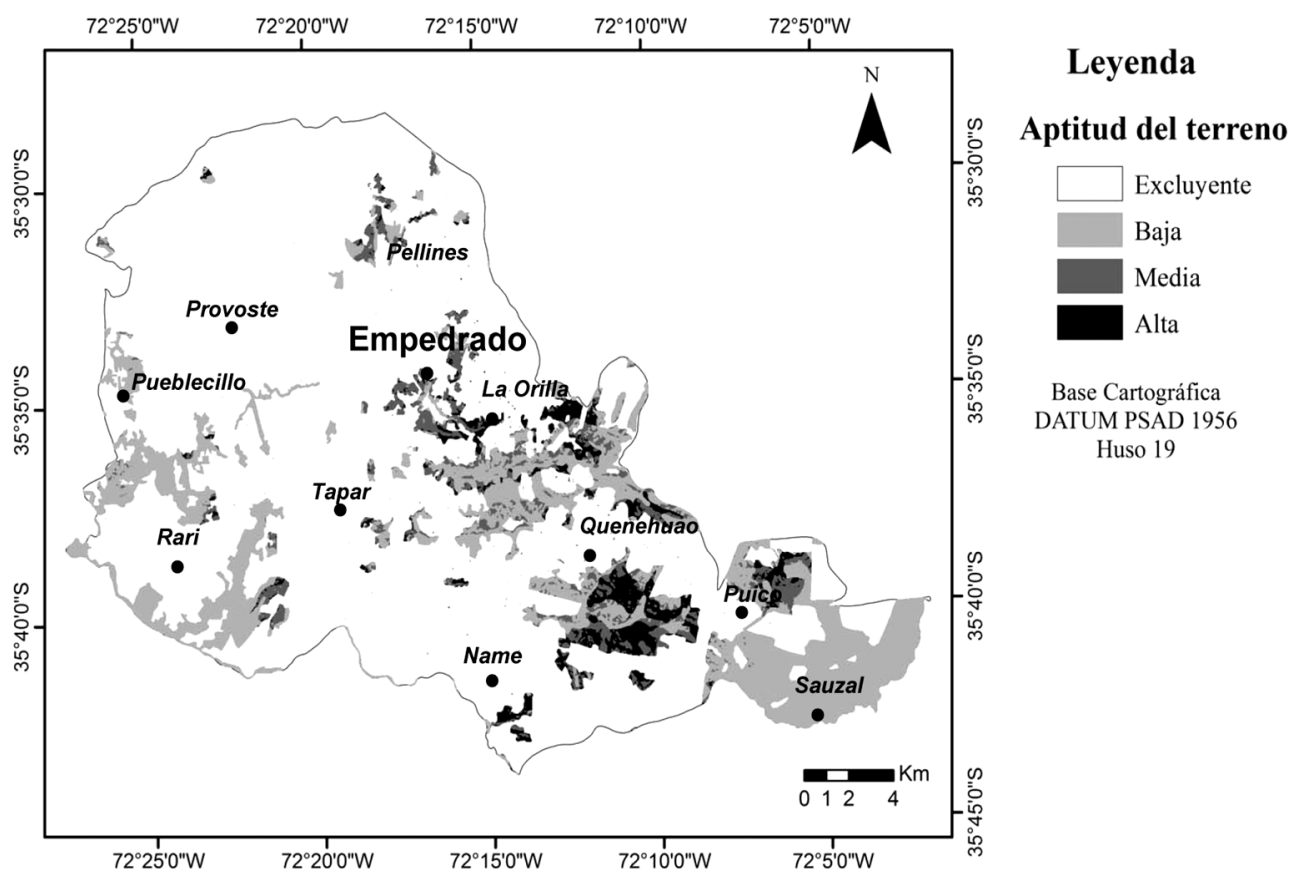

Figura 6. Modelo de capacidad de acogida del territorio para Chamaecytisus proliferus. 


\section{4. Áreas potenciales para Eucalyptus camaldulensis}

Las variables más importantes para determinar áreas potenciales para el establecimiento de Eucalyptus camaldulensis son la temperatura mínima media del mes más frio, la profundidad y el drenaje del suelo mientras que la menos importante es la pendiente del terreno.

$\mathrm{CDA}=0,1424^{*}$ Precipitación $+0,1780^{*} \mathrm{~T}^{\circ}$ mínima $+0,0712^{*} \mathrm{~T}^{\circ}$ máxima + $0,1144^{*}$ Textura $+0,1424 *$ Profundidad $+0,1424 *$ Drenaje $+0,0712 *$ Altitud + $0,0345^{*}$ Pendiente $+0,1036^{*}$ Expos

En la figura 7 se observa que los sectores de la comuna con áreas de alta aptitud corresponden a áreas dispuestas en la sección este de la comuna (calidades media y alta).

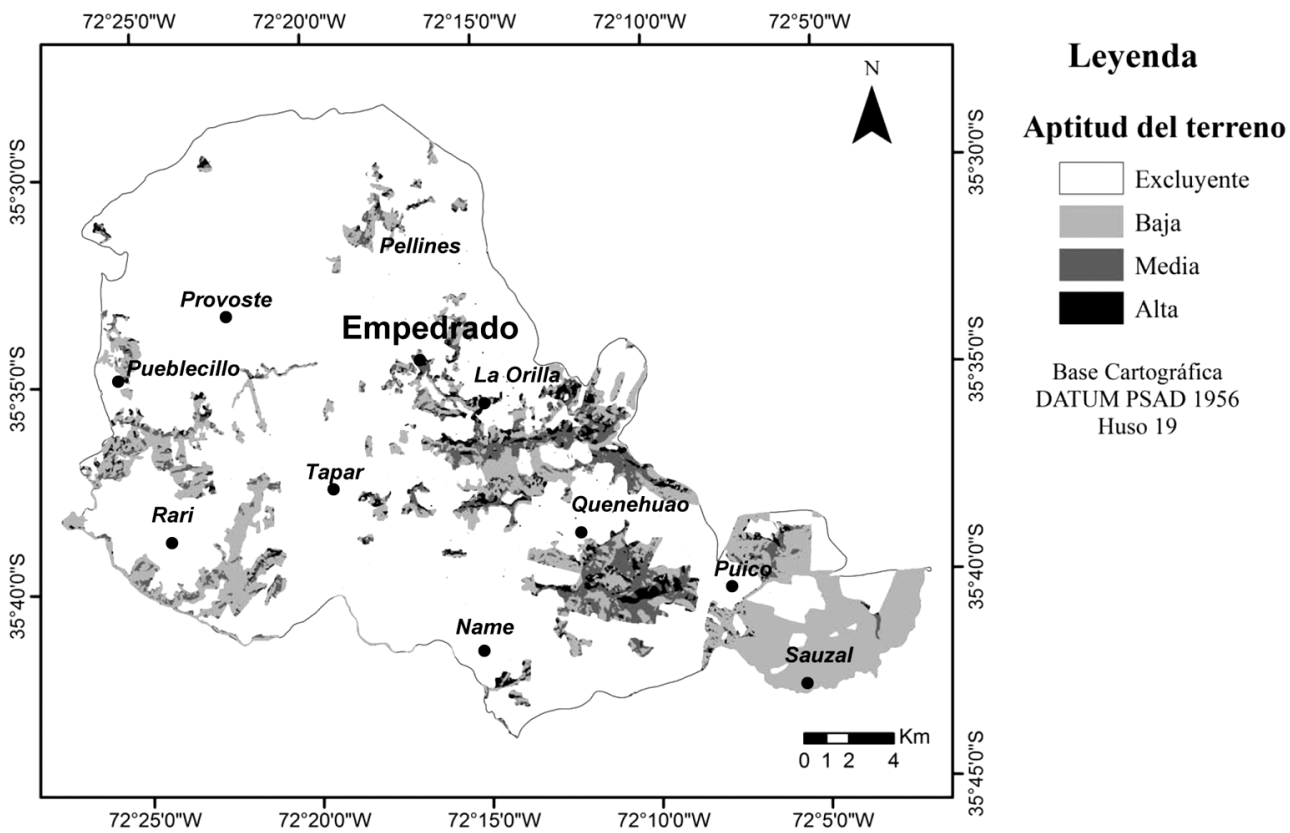

Figura 7. Modelo de capacidad de acogida del territorio para Eucalyptus camaldulensis.

\section{5. Áreas potenciales para Robinia pseudoacacia}

La variable precipitación con un $26.97 \%$ de importancia, es la que más influye en la identificación de áreas potenciales para establecer plantaciones dendroenergéticas de Robinia pseudoacacia. La menos importante es la pendiente con un 3,84\%. 
$\mathrm{CDA}=0,2697^{*}$ Precipitación $+0,0769^{*} \mathrm{~T}^{\circ}$ mínima $+0,0769^{*} \mathrm{~T}^{\circ}$ máxima + $0,1153^{*}$ Textura $+0,1538 *$ Profundidad $+0,0769 *$ Drenaje $+0,0769 *$ Altitud + $0,0384^{*}$ Pendiente $+0,1153^{*}$ Expos

Se observa que el extremo sureste de la comuna no presenta áreas con alta o media aptiud para establecer plantaciones dendroenegéticas de esta especie. Sin embargo esta zona no presenta restricciones de uso en el modelo final de capacidad de acogida (figura 8). Los sectores de la sección sur y este de la comuna son los que concentran las áreas prioritarias para robinia, aunque el modelo de esta especie fue el que obtuvo los menores puntajes de aptitud.

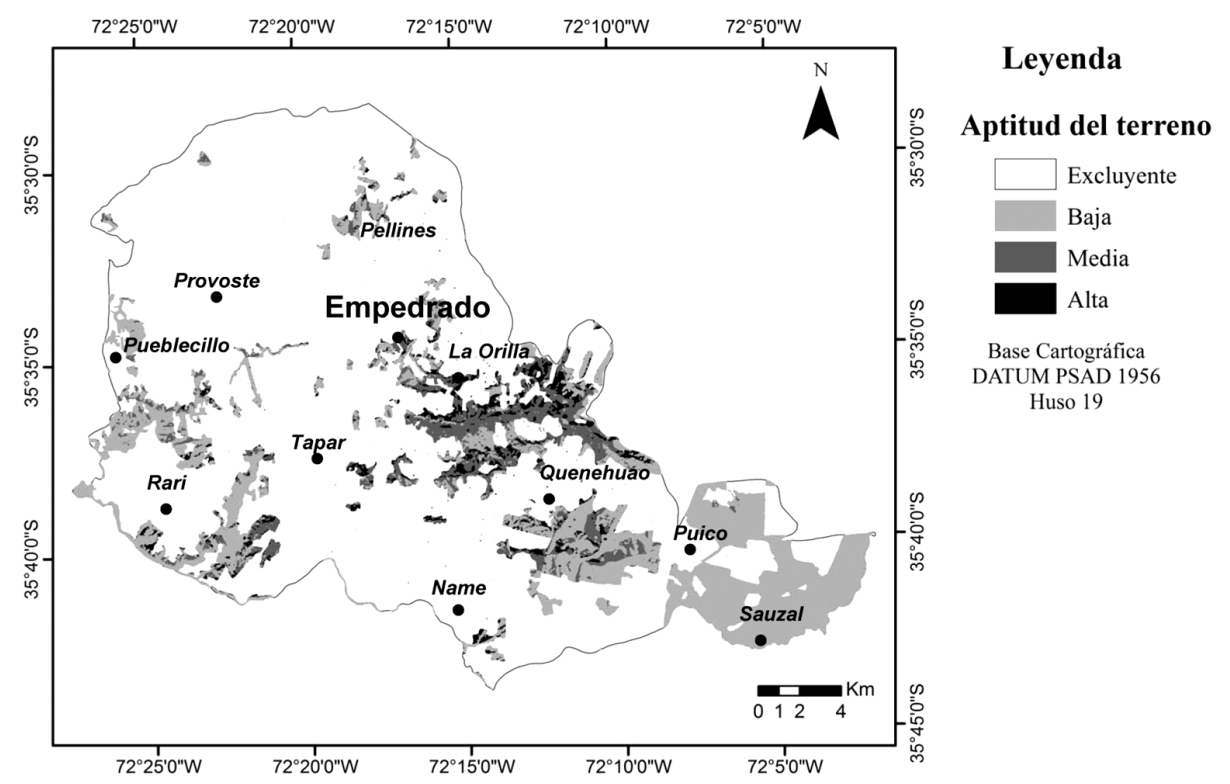

Figura 8. Modelo de capacidad de acogida del territorio para Robinia pseudoacacia.

\subsection{Uso propuesto del suelo}

Al comparar las puntuaciones máximas de los modelos de capacidad de acogida de las especies propuestas (figura 9) se jerarquiza la importancia de una especie sobre otra para un determinado territorio.

- Acacia dealbata domina en el extremo suroeste de la comuna ocupando áreas de baja altitud (menor a $100 \mathrm{msnm}$ ), cercanas a la costa, donde se producen precipitaciones anuales mayores a $900 \mathrm{~mm}$. 
- Acacia melanoxylon se encuentra principalmente en áreas de exposiciones sur, cercanas a cursos de agua, por debajo de $500 \mathrm{msnm}$.

- Chamaecitysus proliferus es la especie que predomina en la mayor parte de la superficie comunal, debido a la gran plasticidad de sus requerimientos ambientales, abarcando sectores de distinta exposición y pendiente, hasta altitudes de $800 \mathrm{msnm}$, con precipitaciones entre 800 a $900 \mathrm{~mm}$.

- Los sectores ubicados en el extremo sureste de la comuna, fueron permanentemente evaluados como de baja aptitud en los modelos de capacidad de acogida. La especie que más se adapta a estas zonas restrictivas en cuanto a variables climáticas y edáficas es Eucalyptus camaldulensis.

- Coincidiendo con modelos de capacidad de acogida como el de Gestión Forestal (2006), Robinia pseudoacacia no aparece como especie recomendable para la comuna de Empedrado en cuanto a uso dendronenergético. Si bien es una especie rústica, generalmente se encuentra asociada a zonas agrícolas abandonadas, llanos o de escasa pendiente y buen drenaje, áreas que en Empedrado son escasas.

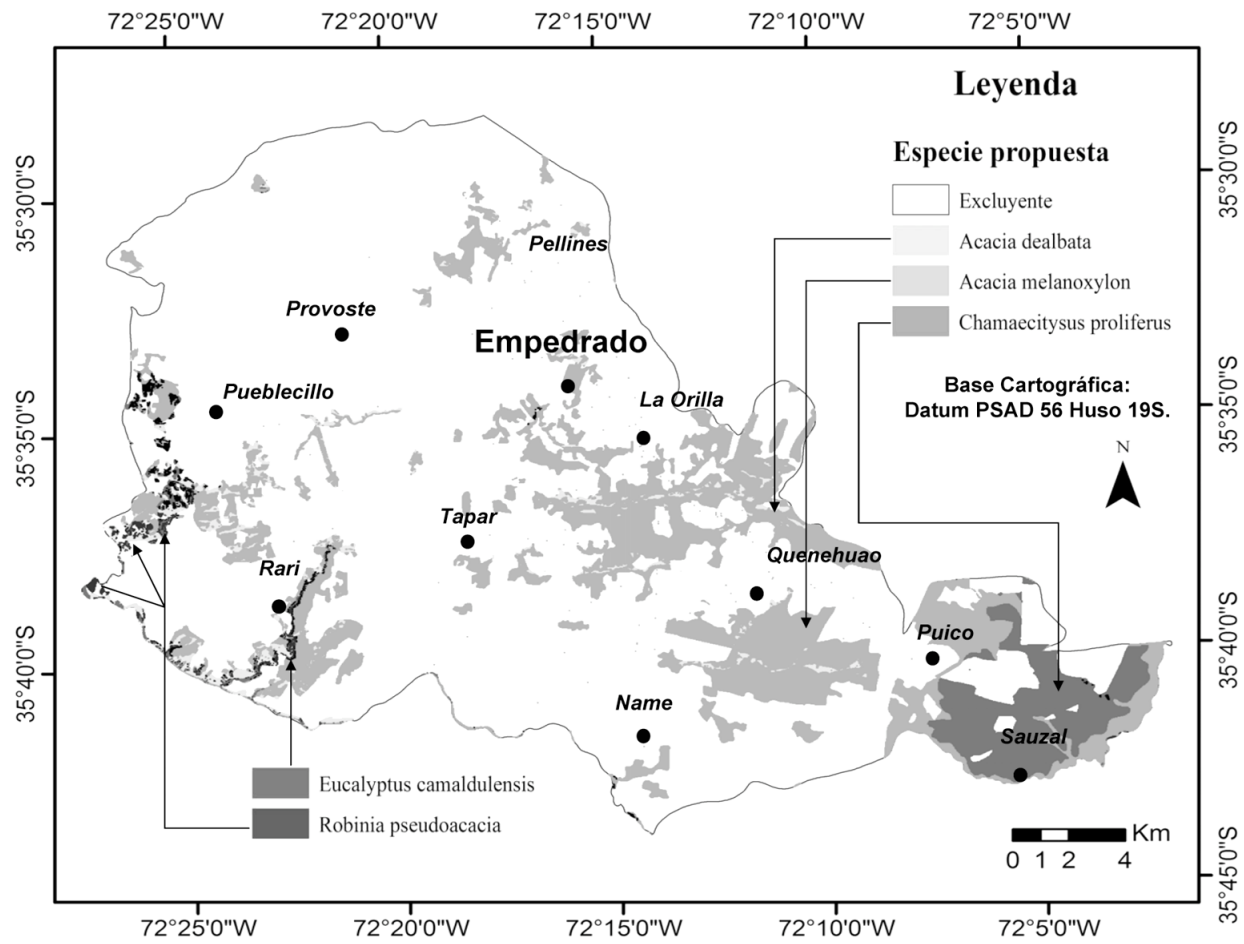

Figura 9. Uso propuesto del suelo para la Comuna de Empedrado. 
Las superficies propuestas para cada especie se presentan en la tabla 2. En orden de preferencia, las especies que presentan mayor aptitud para ser utilizadas en el establecimiento de plantaciones dendroenergéticas en la Comuna de Empedrado, Región del Maule, en un 97,5\% de la superficie potencial disponible actualmente, son Chamaecytisus proliferus, Eucalyptus camaldulensis y Acacia melanoxylon. Un 1,7\% de la superficie disponible para establecer plantaciones presentó las mismas puntuaciones de aptitud para dos especies. Las asociaciones formadas fueron Acacia dealbata - Eucalyptus camaldulensis y Chamaecytisus proliferus - Eucalyptus camaldulensis.

Tabla 2. Superficies propuestas para cada especie.

\begin{tabular}{c|c|c}
\hline Especie & Superficie (Hectáreas) & Porcentaje (\%) \\
\hline Acacia dealbata & 98,8 & 0,7 \\
Acacia melanoxylon & $1.192,7$ & 9,2 \\
Chamaecytisus proliferus & 9.022 & 70,3 \\
Eucalyptus camaldulensis & $2.310,3$ & 18 \\
Robinia pseudoacacia & 0 & 0 \\
A. dealbata - E. camaldulensis & 86,9 & 0,6 \\
C. proliferus - E. camaldulensis & 117,8 & 0,9 \\
\hline
\end{tabular}

\section{Discusión y conclusiones}

Un análisis general de la visión en terreno de los resultados aquí obtenidos, indican que en la práctica las áreas calificadas con alta capacidad de acogida para los distintos tipos de uso del suelo, corresponden prácticamente un 80\% a terrenos que han sido utilizados tradicionalmente para la ganadería y cultivos agrícolas esporádicos en áreas de suelos más profundos y drenados. A su vez la presencia local de incendios en la Comuna de Empedrado, es muy baja, por lo que la existencia de biomasa en las especies propuestas para este estudio no ha variado sustantivamente en los últimos 25 años.

Por tal razón, la Comuna de Empedrado, Región del Maule, posee áreas potenciales físicas para recibir plantaciones forestales con especies para fines de producción de leña. En conjunto se identificaron 12.828,6 hectáreas disponibles actualmente para este uso, y que equivalen al 22,4\% de la superficie comunal. Esta superficie corresponde prácticamente en un 100\% a pequeños y medianos propietarios agrícolas y forestales que vislumbran en este territorio una opción de ingresos económicos a corto y mediano plazo, como alternativa a los actuales sistemas productivos que posee la comuna, basado esencialmente en la ganadería, extracción de leña a menor escala y agricultura de subsistencia. 
Un aspecto importante a considerar en este tipo de estudios es la posibilidad de encontrar conflictos o competencia por usos del suelo que en muchos casos podrían ser incompatibles. Sobre la base de estos resultados, no existe tal conflicto, por cuanto la propuesta territorial de estas especies para su uso en leña, estarían localizándose en áreas ya degradadas por el pastoreo y ganadería, incluso con la opción de recuperar terrenos degradados por la erosión. La Comuna de Empedrado presenta una alta proporción de surcos y cárcavas de erosión, que podrían ser cubiertos con la acogida de estas especies.

En términos específicos, la especie Chamecytisus proliferus aparece como la especie que más se adapta a las condiciones ambientales de la zona de estudio. Eucalyptus camaldulensis aparece como una buena opción en las áreas más degradadas, con restricciones hídricas y edáficas, mientras que Robinia pseudoacacia no es recomendable para establecer plantaciones con fines de producción de leña en Empedrado, considerando variables físicas.

A su vez, el uso del suelo propuesto a través de los modelos de capacidad de acogida del territorio permiten conocer la distribución, superficie y aptitud de los terrenos a forestar con cada especie en particular. Sin embargo estos modelos son simplificaciones de la realidad, por lo que deben usarse como una herramienta de ayuda en la toma de decisiones.

Como comentario final es necesario indicar que la metodología fuzzy aquí propuesta es completamente replicable en territorios de similares características, en cuyo caso el principal cambio a ejecutar es la definición de umbrales de pertenencia y pesos asignados a cada una de las variables. No obstante se trata de un estudio local, es la primera aproximación con una metodología nueva, como alternativa al clásico análisis multicriterio.

\section{Referencias}

Ahmed, T.; Rao, K. y Murthy, J. (2000) GIS based fuzzy membership model for cropland suitability analysis. Agricultural Systems, 63, 75-95.

Álvarez, M. (2003) Localización de sitios adecuados para el establecimiento de plantaciones de quillay (Quillaja saponaria), mediante integración de sistemas de información geográfica y técnicas de evaluación multicriterio. Tesis de grado, Universidad de Talca. $113 \mathrm{p}$.
Astigarraga, E. (2004) El Método Delphi. Facultad de C.C.E.E. y Empresariales. Universidad de Deusto. San Sebastián. http://www.prospectiva.eu/zaharra/Metod O_Delphi.pdf

Barredo, J. (1996) Sistemas de Información Geográfica y Evaluación Multicriterio en la ordenación del territorio. Madrid, Ra-Ma Ed.

Bellman, R. y Zadeh, L. (1970) Decision making in a fuzzy environment. Management Science, 17, 141-164. 
Bonham-Carter G.F. (1994) Geographic Information Systems for geoscientists: modelling with GIS. Pergamon Press.

Burrough, P. (1989) Fuzzy mathematical methods for survey and land evaluation. Journal of Soil Science, 40, 477-492.

Bustillos-Herrera, A.; Valdez-Lazalde, R.; Aldrete, A. y Gonzalez-Guillén, M. (2007) Aptitud de terrenos para plantaciones de Eucalipto (Eucalyptus grandis Hill ex Maiden) Definición mediante el proceso de análisis jerarquizado y SIG http://www.colpos.mx/agrocien/Bimestral /2007/oct-nov/art-8.pdf

Cox, E. (2005) Fuzzy modeling and genetic algorithms for data mining and exploration. Elsevier Inc., USA.

Chen, S. y Hwang, C. (1992) Fuzzy multiple attribute decision-making. Berlin, Springer.

Dubois, D. y Prade, M. (1979) Fuzzy sets and systems: Theory and spplications. New York, Academic Press.

Duprey, B. y Taheri, S.(2010) A fuzzy based stability index using a right sigmoid membership function SAE. International Journal of Commercial Vehicles, 2(2), 177194.
FIA-INDAP-INFOR. (2000) Monografia de Falso Acacio (Robinia pseudoacacia) 54 p.

Gestión Forestal (2006) Tecnología y Gestión Forestal para pequeños y medianos propietarios. Plantaciones. CORFO-INFOR. http://www.gestionforestal.cl/

Gill, A. y Bector, C.R. (1997) A fuzzy linguistic approach to data quantification and construction of distance measures for the part family formation problem. International Journal of Production Research, 13661388, 35(9), 2565-2578.

IGM. Carta Empedrado 1:50.000. (2004) http://www.igm.cl/productoresumen2.asp? $\mathrm{id}=\mathbf{7 7 4}$

Julio, G. (1999) Fundamentos del manejo del fuego. Universidad de Chile. Departamento de Manejo de Recursos Forestales, Santiago.

Konow, I. y Pérez, G. (1990) Métodos y técnicas de investigación prospectiva para la toma de decisiones. http://geocities.com/Pentagon/Quarte rs/7578/pros01.html

Voogd, H. (1983) Multicriteria evaluation for urban and regional planning. Pion, London. 